\title{
BMJ Open Economic evaluation of cognitive behavioural therapy for insomnia (CBT-I) for improving health outcomes in adult population: a systematic review protocol
}

\author{
Andrea Natalie Natsky (D) , ${ }^{1}$ Andrew Vakulin, ${ }^{2,3}$ Ching Li Chai-Coetzer, ${ }^{2,4}$ \\ Leon Lack, ${ }^{2}$ R. Doug McEvoy, ${ }^{2,5}$ Billingsley Kaambwa ${ }^{1}$
}

To cite: Natsky AN, Vakulin A, Chai-Coetzer CL, et al. Economic evaluation of cognitive behavioural therapy for insomnia (CBT-I) for improving health outcomes in adult population: a systematic review protocol. BMJ Open 2019;9:e032176. doi:10.1136/ bmjopen-2019-032176

- Prepublication history and additional material for this paper are available online. To view these files, please visit the journal online (http://dx.doi org/10.1136/bmjopen-2019032176).

Received 06 June 2019 Revised 26 September 2019 Accepted 09 October 2019

Check for updates

(C) Author(s) (or their employer(s)) 2019. Re-use permitted under CC BY-NC. No commercial re-use. See rights and permissions. Published by BMJ.

For numbered affiliations see end of article.

Correspondence to Andrea Natalie Natsky; andrea.natsky@flinders.edu.au

\section{ABSTRACT}

Introduction Insomnia is associated with a number of adverse consequences that place a substantial economic burden on individuals and society. Cognitive behavioural therapy for insomnia (CBT-I) is a promising intervention that can improve outcomes in people who suffer from insomnia. However, evidence of its cost-effectiveness remains unclear. In this study, we will systematically review studies that report on economic evaluations of CBT-I and investigate the potential economic benefit of CBT-I as a treatment for insomnia.

Methods and analysis The search will include studies that use full economic evaluation methods (ie, costeffectiveness, cost-utility, cost-benefit, cost-consequences and cost-minimisation analysis) and those that apply partial economic evaluation approaches (ie, cost description, cost-outcome description and cost analysis). We will conduct a preliminary search in MEDLINE, Google Scholar, MedNar and ProQuest dissertation and theses to build the searching terms. A full search strategy using all identified keywords and index terms will then be undertaken in several databases including MEDLINE, Psychinfo, Proquest, Cochrane, Scopus, Cumulative Index of Nursing and Allied Health Literature (CINAHL), Web of Science and EMBASE. We will adhere to the Preferred Reporting Items for Systematic Reviews and MetaAnalyses for protocol guidelines in this review. Only articles in the English language and those reporting on adult populations will be included. We will use standardised data extraction tools for economic evaluations to retrieve and synthesise information from selected studies into themes and summarised in a Joanna Briggs Institute dominance ranking matrix.

Ethics and dissemination No formal ethics approval will be required as we will not be collecting primary data. Review findings will be disseminated through a peer-reviewed publication, workshops, conference presentations and a media release.

PROSPERO registration number CRD42019133554.

\section{BACKGROUND}

Insomnia is characterised by difficulties in initiating or maintaining sleep and/or early

\section{Strengths and limitations of this study}

- This systematic review will use a comprehensive search strategy in order to capture all available evidence of economic evaluation studies of cognitive behavioural therapy for insomnia (CBT-I).

- This review will use standardised tools to extract, synthesise and appraise studies in order to provide high-level evidence of the value for money of CBT-I.

- This review primarily intends to include both full and partial economic evaluation studies of CBT-I.

- Studies that will be included in the review will be limited to English and adult population, which could lead to information bias.

morning awakenings despite sufficient opportunities for sleep, which results in poor sleep quality and daytime impairment. ${ }^{1}$ Depending on the terminology used to define and diagnose insomnia, its prevalence can range from $3.9 \%$ to $22.1 \%$, making it one of the most common sleep disorders among adults. ${ }^{2-5}$ The high prevalence and debilitating nature of insomnia contribute to the substantial health and economic burden. Insomnia is associated with a range of adverse health consequences for individuals, including poor daytime function and reduced health-related quality of life (HRQoL). ${ }^{6} 7$ Consequently, people suffering from insomnia tend to have lower work productivity, absenteeism, an increased risk of motor vehicle accidents and future psychiatric disorders (eg, major depressive disorder), ${ }^{8}$ thereby increasing healthcare utilisation and economic costs. ${ }^{7-11}$

A Canadian study by Daley et $a l^{12}$ estimated the total direct and indirect cost of insomnia to be Can $\$ 6.6$ billion per annum or equal to $1 \%$ of the gross domestic product of Quebec province. Of this cost, $96 \%$ was attributable 
to indirect costs (presenteeism ( $\$ 5.0$ billion) and absenteeism (\$970.6million)), and direct expenditure of alcohol use as a sleep aid (\$339.8 million). Reynolds and Ebben ${ }^{13}$ estimated that the direct cost of insomnia contributes to approximately $4 \%-16.7 \%$ of the total US and Canadian national economic cost based on studies done in the two countries. ${ }^{12} 14$ Furthermore, the direct cost resulting from hospitalisation, other health services utilisation, prescription and over-the counter medicines are also substantial. ${ }^{15}$ Walsh and Engelhardt ${ }^{16}$ estimated the direct cost of insomnia in the USA in 1990 to be about US $\$ 10.9$ billion, of which almost $90 \%$ originated from nursing home care and $10 \%$ was for prescribed medications. Reynolds and Ebben ${ }^{13}$ reanalysed data from Stoller ${ }^{14}$ and reported that the total direct and indirect annual expenditure of insomnia in 2016 was between US $\$ 150.36$ and US $\$ 174.89$ billion after adjusting for inflation. In Australia, total health system expenditure attributed to insomnia in 2010 was estimated to be $\$ 118.7$ million, while the indirect financial cost amounted to $\$ 1.5$ billion $^{17}$. From this earlier estimate, it is evident that insomnia is associated with substantial societal economic costs as derived by aggregating all direct and indirect costs borne by society. Unfortunately, it is difficult to estimate the true financial cost because most of the expenses are made up of indirect costs. The non-uniform reporting of insomnia-related expenses between studies adds to this difficulty.

In order to reduce the excessive costs of insomnia, it is essential to use interventions that can improve symptoms and reduce the economic burden. Cognitive behavioural therapy for insomnia (CBT-I) is an intervention that aims to change dysfunctional thought and behaviour patterns through a combination of several components including stimulus control, sleep-restriction therapy, cognitive therapy for dysfunctional thoughts, sleep hygiene, sleep education and relaxation techniques. ${ }^{513}{ }^{18-21}$ Numerous meta-analyses have demonstrated the effectiveness of CBT-I in samples with isolated insomnia or insomnia co-occurring with specific co-morbidities; in specialist care, primary care, and community settings; and with different CBT-I modalities including individualised, group and online treatments. ${ }^{22-29}$ CBT-I is usually delivered over 4-8 weekly sessions; yet its effect in enhancing sleep quality and quantity can be sustained for up to 3 years with minimum risk compared with pharmacotherapy. ${ }^{23}{ }^{30}$ The sustained improvements in insomnia achieved by CBT-I are attributed to the fact that it specifically addresses the key underlying and perpetuating dysfunctional behaviours, cognitions and conditioned relationships. ${ }^{23}{ }^{30}$ Although the effectiveness of CBT-I is widely accepted and is recommended as a first-line treatment in the clinical guidelines of several countries, ${ }^{431-34}$ research that report on its economic benefits is still limited.

Economic evaluation is defined as the comparative assessment of the cost and benefits of alternative interventions, or between an assessed intervention and its comparator(s). ${ }^{35}$ The five main types of economic evaluation are cost-effectiveness analysis (CEA), cost minimisation analysis, cost utility analysis (CUA), cost benefit analysis $(\mathrm{CBA})$ and cost consequences analysis (CCA). ${ }^{35}$ Despite the abundance of economic evaluation applications in the healthcare sector, there is a critical gap in economic evaluation evidence for CBT-I. ${ }^{36}$ Previous reviews ${ }^{37} 38$ have acknowledged the lack of studies investigating economic impact of insomnia interventions and argued that treating insomnia costs less than not treating the condition. Since then, there have been a growing number of economic studies that also incorporate a novel delivery mechanism of CBT-I through an online platform. ${ }^{39}$ We propose to capture the recent and best available evidence focusing on the application of economic evaluation of CBT-I, provide a critical appraisal of such evidence and assess the potential economic benefit of this intervention in the treatment of insomnia. Importantly, no systematic review is currently available that has synthesised this information. Hence, we aim to update existing literature in this area by conducting a structured systematic review and utilising standard reporting of economic studies. Our systematic review and accompanying metaanalysis of evidence address this knowledge gap and will generate information that will help decision-makers determine whether implementing CBT-I as first-line insomnia treatment provides value for money. Systematic reviews that demonstrate the cost-effectiveness of interventions help inform policymakers and facilitate the dissemination and implementation of effective treatments for insomnia and other areas of sleep health.

\section{METHODS AND DESIGN \\ Inclusion criteria}

Population

The population of interest will be the general adult population (individuals aged 18 years or older) who suffer from insomnia.

\section{Intervention of interest and comparator}

The target intervention is CBT-I. This review will include all studies that incorporate at least one of the five most widely accepted components of CBT-I, that is, stimulus control, sleep restriction, cognitive therapy, sleep hygiene and relaxation therapy. Studies of all modalities (eg, face-to-face individual/group, telephone, online) will be included.

This review will consider studies that compared the intervention to control groups. The control groups will include individuals who received pharmacotherapy, alternative therapies or non-evidence-based treatments to treat insomnia or those who receive no treatment.

Context

There will be no restriction as to where the studies took place. Studies conducted in primary healthcare, specialist 
care and online services of high and low resource settings will be considered for inclusion in the review.

\section{Costs and outcome measures}

This review will report on the estimates of resource use, costs, effectiveness and cost-effectiveness of CBT-I in its natural units or as per corresponding studies. The findings regarding cost-effectiveness of the intervention will be reported in the form of cost per unit of effect expressed in clinical outcome units for CEA and CCA for example, reduction in insomnia symptom based on a specific instrument such as insomnia severity index (ISI) scores. ${ }^{40}$ While for CUA, the findings will be reported as cost per a summarised measure of impact on HRQoL valued as utility such as cost per quality adjusted life years (QALYs) or disability adjusted life years (DALYs). QALYs are a combination of life expectancy and the quality of life during the survival time which is calculated by multiplying utility or HRQoL by survival time while DALYs quantify the burden of disease by adding years of life lost due to premature mortality and years lived in disability. ${ }^{35} 41$ The findings of CCA, CEA and CUA can be summarised in the form of an incremental cost-effectiveness ratio (ICER) that is usually the main output or result of economic evaluation studies. The ICER is generated by dividing the difference in total cost (incremental cost) by the difference in health outcome (incremental effect) that could be QALY, DALY or ISI score relative to study design. ${ }^{35}$ Furthermore, this review will report on cost-effectiveness acceptability curve (CEAC) for relevant studies that particularly did not report on the measures outlined earlier. The CEAC is a graph that displays the probability that an intervention will be the cost-effective option at different willingness to pay thresholds. On the other hand, findings from CBA will be in a form of net benefit ratio which is a measure, in monetary terms, of the health and nonhealth benefits associated with an intervention relative to its costs. ${ }^{35}$ Assigning a dollar value to health outcomes can be achieved in various ways including through the use of willingness to pay and human capital approaches. ${ }^{35}$ In the case where CBT-I is integrated with other interventions/ programme (eg, combination of CBT-I and pharmacotherapy) and data of reported measures are not available separately, the reviewers will analyse measures of interest for CBT-I in combination with others.

A societal cost perspective is the broadest viewpoint as it accounts for all costs to society as a whole regardless of which party bears the cost and is the preferred viewpoint for this review. ${ }^{35}$ Nonetheless, this review will also report on other cost perspectives including those of individual patient and third-party payers (eg, Ministry of Health, public sector, government, national insurance).

\section{Study design}

The systematic review will consider partial (eg, costdescription studies, cost analysis and cost-outcome descriptions) and full economic evaluation studies (CUA, CBA, CCA and CEA) that assess CBT-I versus comparators.
Both model and non-model-based economic evaluations will be considered.

\section{Language}

Only studies published in English before June 2019.

\section{Exclusion criteria}

Studies will be excluded if they report on populations that include non-human subjects or children and/or adolescents $(<18$ years). In addition, the following will also be excluded: theory papers, letters, editorials, reviews, theses or dissertations and studies where full texts could not be obtained.

\section{Search strategy}

We will perform the following steps as part of the search strategy that aims to find economic evaluation studies on CBT-I. First, a preliminary limited search through MEDLINE will be undertaken as well as analysis of the text words contained in the title and abstract and of the index terms used to describe articles. The initial search will also include grey literature from ProQuest dissertation and theses and other health data repositories such as MedNar and Google Scholar. The first step is crucial in order to develop key terms for three key pre-defined concepts relating to the research question as follows:

Concept 1 (insomnia, sleep initiation and maintenance disorder, sleep wake disorders, sleep disorders, sleep problem, sleep complaint, sleep disturbance, poor sleep); concept 2 (cognitive behavio* therapy for insomnia, CBT-I, bed restriction, relaxation therapy, stimulus control, cognitive therapy, biofeedback, sleep hygiene, mindfulness therapy, sleep habit therapy, relaxation therapy, nonpharma*); concept 3 (economic evaluation, economics, QALY, quality of life, value of life, cost and cost analysis, cost benefit, cost utility, cost effectiveness, healthcare cost, economic model, economic analysis, cost minimisation)

Second, we will conduct a full search (see online supplementary appendix 1) using all identified keywords and index terms across the following databases: MEDLINE, Psychinfo, Proquest, Cochrane, Scopus, Cumulative Index of Nursing and Allied Health Literature (CINAHL), Web of Science and EMBASE. Concepts 1,2 and 3 will be connected by 'AND' to run the full search strategy in the databases mentioned previously.

Third, relevant titles and abstracts from each database will be screened and two reviewers will select eligible studies for full text appraisal. The final step will include backward and forward citation-chaining of relevant literature from the reference lists of all identified reports and articles. Studies published in English and before June 2019 will be considered for inclusion in this review.

The schematic presentation of the search strategy using the Preferred Reporting Items for Systematic Reviews and Meta-Analyses for protocol guidelines is presented in figure 1 . 
1. Preliminary search from Google Scholar and MEDLINE using concepts including insomnia, cognitive behaviour therapy for insomnia, economic evaluations

2. Analyse text words to build full search strategy

3. Conduct full search using the finalised search strategy derived from identified concepts

4. Replicate full search in several databases such as MEDLINE, Psychinfo, Proquest, Cochrane, Scopus, CINAHL, Web of Science and EMBASE.

5. Apply study restriction to English but not to dates

6. Screen studies for eligibility according to inclusion and exclusion criteria

7. Additional studies to be included from reference list of relevant articles

8. Oblige to PRISMA guidelines

Figure 1 A systematic study selection process.

\section{Study selection}

Two independent reviewers will assess selected documents using the inclusion and exclusion criteria before including a study in the review. Any disagreement that may arise will be resolved through research team consensus.

\section{Data extraction and synthesis}

Data will be extracted from relevant papers included in the review using the Joanna Briggs Institute (JBI) Data Extraction Form for Economic Evaluations (see online supplementary appendix 2). ${ }^{42}$ The extracted data will encompass primarily three sections which include descriptive data, results and author conclusions. The first section covers description of three sets of items: (1) study population, intervention, comparator(s) and outcomes; (2) study methods including economic evaluation study design type, analytic perspective(s), source of effectiveness data, prices and currency used for costing, analytical duration/period, sensitivity analysis, cost-effectiveness method and measures of resource use, cost and health outcome; (3) study setting (geographical location, healthcare and other service delivery setting). This will be followed by the second section that reports on the studies' results for resource usage, cost and cost-effectiveness measures. Finally, the authors will discuss factors that promote cost-effectiveness of the intervention of interest (CBT-I) based on comparative findings of the included studies and other relevant economic research.
The nature of data extraction and synthesis will depend on the quality, quantity and contexts of the identified economic studies. Where possible, economic findings (mainly differences in incremental costs and outcomes between intervention and comparator groups) will be synthesised using meta-analysis in Stata V.13.1. ${ }^{43}$ Binary outcome data will be reported as ORs with $95 \%$ CI, while mean differences (MD) and 95\% CI will be reported for continuous data. Estimates of OR and MD will be calculated using appropriate standardised methods (eg, the Mantel-Haenszel ${ }^{44}$ method for binary outcomes and random-effect or fixed-effect models $^{45}$ for continuous data). Heterogeneity will be assessed using $\mathrm{I}^{2}$ statistics, with a value of $85 \%$ or more signifying considerable heterogeneity. ${ }^{46}$ Healthcare costs will be reported in 2019 Australian Dollars after conversion and adjustment for inflation where relevant. However, if data do not permit for pooling of results, qualitative analyses using the JBI dominance ranking Matrix ${ }^{42}$ which displays three-by-three matrix with three classifications (strong dominance, weak dominance and non-dominance (online supplementary appendix 3)), will be performed.

\section{Risk of bias (quality) assessment}

Two reviewers will independently assess the methodological quality and validity of the relevant economic evaluation studies using the standardised JBI Critical Appraisal Checklist for Economic Evaluation ${ }^{47}$ that is based on the guidelines developed by Drummond et al. ${ }^{35}$ For economic evaluation using modelling study design, an additional checklist will be utilised based on the critical checklist for modelling developed by Philips et al. ${ }^{48}$ Given the variety of settings and type of evaluations of potential included studies, the European Network of Health Economic Evaluation Databases checklist will be applied to further assess transferability and generalisability of the studies to their setting of interest. ${ }^{49}$ The reviewers will individually assess each selected study for any risk of bias therein using the aforementioned tools. ${ }^{47-49}$

\section{Ethics and dissemination}

No formal ethical approval is required as this study will not involve collection of primary data. Findings will be disseminated in the following media: publication in peerreviewed journals, presentation at conferences, workshops and sharing through a media release.

\section{Patient and public involvement}

There is no patient or public involvement in the design or planning of this review.

\section{DISCUSSION}

This systematic review will provide evidence relating to economic evaluation of CBT-I across a range of settings using a reproducible and transparent procedure for systematic literature searching. This protocol 
has outlined detailed inclusion and exclusion criteria of eligible studies, which include a description of the target population, intervention of interest and comparator, context, study design, cost and outcome measures, as well as database sources, search strategy, data extraction and synthesising method. Using comprehensive and standardised tools to appraise such evidence, this study will assess the quality of available studies in relation to the resource use, cost and cost-effectiveness of CBT-I. Potential limitations of this review include the scarcity of evidence and heterogeneity of measures, cost perspective and outcomes evaluated which could negatively impact on the transferability and generalisability of the data synthesis. Studies that will be included in the review will be limited to those reported in the English language and in adult populations, which could also lead to information bias.

As we are facing resource constraints and rising costs in the current healthcare climate, evidence-based guidance regarding cost-effectiveness of CBT-I can potentially help to inform decision making in relation to the choice of first-line treatment of insomnia. This review will also identify gaps in the evidence base and offer recommendations for future research priorities in this area.

\section{Author affiliations}

${ }^{1}$ Department of Health Economics, College of Medicine and Public Health, Flinders University, Bedford Park, South Australia, Australia

${ }^{2}$ Adelaide Institute for Sleep Health: A Flinders Centre for Research Excellence, Flinders University, Adelaide, South Australia, Australia

${ }^{3}$ NeuroSleep and Woolcock Institute of Medical Research, University of Sydney, Camperdown, New South Wales, Australia

${ }^{4}$ Sleep Health Service, Southern Adelaide Local Health Network, SA Health, Bedford Park, South Australia, Australia

${ }^{5}$ Department of Medicine, Flinders University, Adelaide, South Australia, Australia

Acknowledgements We thank Flinders University for enabling us to access articles that are not freely available. Special thanks to Ms Leila Mohammadi who helped with the database search strategy. We also acknowledge authors of primary studies.

Contributors BK was responsible for study conception. ANN developed the search strategy and wrote the first draft of the manuscript. ANN, BK, AV and CLC-C contributed to the development of the selection criteria, the risk of bias assessment strategy and data extraction criteria. AV, CLC-C, LL and DM provided expertise on insomnia and CBT-I. All authors have read, provided feedback, revised and approved the final manuscript.

Funding The authors would like to acknowledge funding support from the Australian Government Research Training Program and National Health and Medical Research Council (NHMRC): Clinical Research Excellence (CRE), National Centre of Sleep Health Services Research.

Competing interests None declared.

Patient consent for publication Not required.

Provenance and peer review Not commissioned; externally peer reviewed.

Open access This is an open access article distributed in accordance with the Creative Commons Attribution Non Commercial (CC BY-NC 4.0) license, which permits others to distribute, remix, adapt, build upon this work non-commercially, and license their derivative works on different terms, provided the original work is properly cited, appropriate credit is given, any changes made indicated, and the use is non-commercial. See: http://creativecommons.org/licenses/by-nc/4.0/.

\section{ORCID iD}

Andrea Natalie Natsky http://orcid.org/0000-0002-5290-754X
REFERENCES

1 American Academy of Sleep Medicine. International classification of sleep disorders. 3rd edn. Darien, IL: American Academy of Sleep Medicine, 2014.

2 Chung K-F, Yeung W-F, Ho FY-Y, et al. Cross-Cultural and comparative epidemiology of insomnia: the diagnostic and statistical manual (DSM), International classification of diseases (ICD) and international classification of sleep disorders (ICSD). Sleep Med 2015;16:477-82.

3 National Institutes of Health. National Institutes of health state of the science conference statement on manifestations and management of chronic insomnia in adults, June 13-15, 2005. Sleep 2005;28:1049-57.

4 Qaseem A, Kansagara D, Forciea MA, et al. Management of chronic insomnia disorder in adults: a clinical practice guideline from the American College of physicians. Ann Intern Med 2016;165:125-33.

5 Schutte-Rodin S, Broch L, Buysse D, et al. Clinical guideline for the evaluation and management of chronic insomnia in adults. J Clin Sleep Med 2008;4:487-504.

6 Fullerton DSP. The economic impact of insomnia in managed care: a clearer picture emerges. Am J Manag Care 2006;12:S246-52.

7 Wade AG. The societal costs of insomnia. Neuropsychiatr Dis Treat 2010;7:1-18.

8 Staner L. Comorbidity of insomnia and depression. Sleep Med Rev 2010;14:35-46.

9 Léger D, Bayon V. Societal costs of insomnia. Sleep Med Rev 2010;14:379-89.

10 Deloitte Access Economics. Asleep on the job: costs of inadequate sleep in Australia, report for the sleep health Foundation Australia; 2017.

11 Wickwire EM, Tom SE, Scharf SM, et al. Untreated insomnia increases all-cause health care utilization and costs among Medicare beneficiaries. Sleep 2019;42. doi:10.1093/sleep/zsz007. [Epub ahead of print: 01 Apr 2019].

12 Daley M, Morin CM, LeBlanc M, et al. The economic burden of insomnia: direct and indirect costs for individuals with insomnia syndrome, insomnia symptoms, and good sleepers. Sleep 2009;32:55-64.

13 Reynolds SA, Ebben MR. The cost of insomnia and the benefit of increased access to evidence-based treatment: cognitive behavioral therapy for insomnia. Sleep Med Clin 2017;12:39-46.

14 Stoller MK. Economic effects of insomnia. Clin Ther 1994;16:873-97; discussion 854.

15 Ozminkowski RJ, Wang S, Walsh JK. The direct and indirect costs of untreated insomnia in adults in the United States. Sleep 2007;30:263-73.

16 Walsh JK, Engelhardt CL. The direct economic costs of insomnia in the United States for 1995. Sleep 1999;22 Suppl 2:S386-93.

17 Deloitte Access Economics. Re-awakening Australia: the economic cost of sleep disorders in Australia, 2010, report for the sleep health Foundation Australia; 2011.

18 Ebben MR, Narizhnaya M. Cognitive and behavioral treatment options for insomnia. Mt Sinai J Med 2012;79:512-23.

19 Bootzin RR. Stimulus control for the treatment of insomnia. Proceedings of the American Psychological Association, 1972:395-6.

20 Spielman AJ, Saskin P, Thorpy MJ. Treatment of chronic insomnia by restriction of time in bed. Sleep 1987;10:45-56.

21 Harvey AG. A cognitive model of insomnia. Behav Res Ther 2002;40:869-93.

22 Geiger-Brown JM, Rogers VE, Liu W, et al. Cognitive behavioral therapy in persons with comorbid insomnia: a meta-analysis. Sleep Med Rev 2015;23:54-67.

23 Okajima I, Komada Y, Inoue Y. A meta-analysis on the treatment effectiveness of cognitive behavioral therapy for primary insomnia. Sleep Biol Rhythms 2011;9:24-34.

24 Smith MT, Perlis ML, Park A, et al. Comparative meta-analysis of pharmacotherapy and behavior therapy for persistent insomnia. Am $J$ Psychiatry 2002;159:5-11.

25 Cheng SK, Dizon J. Computerised cognitive behavioural therapy for insomnia: a systematic review and meta-analysis. Psychother Psychosom 2012;81:206-16.

26 Koffel EA, Koffel JB, Gehrman PR. A meta-analysis of group cognitive behavioral therapy for insomnia. Sleep Med Rev 2015;19:6-16.

27 Seyffert M, Lagisetty P, Landgraf J, et al. Internet-Delivered cognitive behavioral therapy to treat insomnia: a systematic review and metaanalysis. PLoS One 2016;11:e0149139.

28 Wu JQ, Appleman ER, Salazar RD, et al. Cognitive behavioral therapy for insomnia comorbid with psychiatric and medical conditions: a meta-analysis. JAMA Intern Med 2015;175:1461-72. 
29 Zachariae R, Lyby MS, Ritterband LM, et al. Efficacy of internetdelivered cognitive-behavioral therapy for insomnia - A systematic review and meta-analysis of randomized controlled trials. Sleep Med Rev 2016;30:1-10.

30 Backhaus J, Hohagen F, Voderholzer U, et al. Long-Term effectiveness of a short-term cognitive-behavioral group treatment for primary insomnia. Eur Arch Psychiatry Clin Neurosci 2001;251:35-41.

31 Morgenthaler T, Kramer M, Alessi C, et al. Practice parameters for the psychological and behavioral treatment of insomnia: an update. An American Academy of sleep medicine report. Sleep 2006;29:1415-9.

32 Ree M, Junge M, Cunnington D. Australasian sleep association position statement regarding the use of psychological/behavioral treatments in the management of insomnia in adults. Sleep Med 2017;36 Suppl 1:S43-7.

33 Riemann D, Baglioni C, Bassetti C, et al. European guideline for the diagnosis and treatment of insomnia. J Sleep Res 2017;26:675-700.

34 Wilson SJ, Nutt DJ, Alford C, et al. British association for psychopharmacology consensus statement on evidence-based treatment of insomnia, parasomnias and circadian rhythm disorders. J Psychopharmacol 2010;24:1577-601.

35 Drummond MF, Sculphe MJ, Torrance GW, et al. Methods for the economic evaluation of health care programs. New York: Oxford University Press, 2015.

36 Mohit B, Cohen JT. Trends of cost-effectiveness studies in sleep medicine. Sleep Med 2019;53:176-80.

37 Botteman M. Health economics of insomnia therapy: implications for policy. Sleep Med 2009;10:S22-5.

38 Wickwire EM, Shaya FT, Scharf SM. Health economics of insomnia treatments: the return on investment for a good night's sleep. Sleep Med Rev 2016;30:72-82.
39 Thiart H, Ebert DD, Lehr D, et al. Internet-Based cognitive behavioral therapy for insomnia: a health economic evaluation. Sleep 2016;39:1769-78.

40 Bastien C, Vallieres A, Morin CM. Validation of the insomnia severity index as an outcome measure for insomnia research. Sleep Med 2001;2:297-307.

41 Sassi F. Calculating QALYs, comparing QALY and DALY calculations. Health Policy Plan 2006;21:402-8.

42 The Joanna Briggs Institute. Joanna Briggs Institute Reviewers' Manual: 2014 edition / Supplement. Adelaide, South Australia: The Joanna Briggs Institute, 2014.

43 StataCorp L. Stata: release 13-statistical software. TX: College Station, 2013.

44 Mantel N, Haenszel W. Statistical aspects of the analysis of data from retrospective studies of disease. $J$ Nat/ Cancer Inst 1959;22:719-48.

45 Cooper H. Research synthesis and meta-analysis: a step-by-step approach. Sage publications, 2015.

46 Leonarde-Bee J. Presenting and interpreting meta-analyses: heterogenity school of nursing and academic division of midwifery University of Nottingham; 2007.

47 Gomersall JS, Jadotte YT, Xue Y, et al. Conducting systematic reviews of economic evaluations. Int J Evid Based Healthc 2015;13:170-8

48 Philips Z, Ginnelly L, Sculpher M, et al. Review of guidelines for good practice in decision-analytic modelling in health technology assessment. Health Technol Assess 2004;8:1-158.

49 Nixon J, Rice S, Drummond M, et al. Guidelines for completing the EURONHEED transferability information checklists. Eur $J$ Health Econ 2009;10:157-65. 